\title{
Existence of global weak solutions to the Navier-Stokes equations in weighted spaces
}

\author{
Zachary Bradshaw, Igor Kukavica, and Tai-Peng Tsai
}

October 16, 2019

\author{
Department of Mathematics, University of Arkansas, Fayetteville, AR 72701 \\ e-mail: zb002@uark.edu \\ Department of Mathematics, University of Southern California, Los Angeles, CA 90089 \\ e-mail: kukavica@usc.edu \\ Department of Mathematics, University of British Columbia, Vancouver, BC V6T 1 Z2 \\ e-mail: ttsai@math.ubc.ca
}

\begin{abstract}
We obtain a global existence result for the three-dimensional Navier-Stokes equations with a large class of data allowing growth at spatial infinity. Namely, we show the global existence of suitable weak solutions when the initial data belongs to the weighted space $\dot{M}_{\mathcal{C}}^{2,2}$ introduced in [2]. This class is strictly larger than currently available spaces of initial data for global existence and includes all locally square integrable discretely selfsimilar data. We also identify a sub-class of data for which solutions exhibit eventual regularity on a parabolic set in space-time.
\end{abstract}

\section{Introduction}

The Navier-Stokes equations describe the evolution of the velocity $u$ and the pressure $p$, solving

$$
\begin{aligned}
& \partial_{t} u-\Delta u+u \cdot \nabla u+\nabla p=0, \\
& \nabla \cdot u=0,
\end{aligned}
$$

in the sense of distributions $[6,11,27,21,23,29,30]$. The system $(1.1)$ is set on $\mathbb{R}^{3} \times(0, T)$ where $T>0$ can be $+\infty$. Also, $u$ evolves from a prescribed, divergence-free initial data $u_{0}: \mathbb{R}^{3} \rightarrow \mathbb{R}^{3}$.

In [24], J. Leray constructed a global-in-time weak solution to $(1.1)$ on $\mathbb{R}^{3} \times(0, \infty)$ for any divergence-free vector field $u_{0} \in L^{2}\left(\mathbb{R}^{3}\right)$. In [21], Lemarié-Rieusset introduced a local analogue of a Leray weak solution evolving from uniformly locally square integrable data $u_{0} \in L_{\text {uloc }}^{2}$. Here, $L_{\text {uloc }}^{q}$, for $1 \leq q \leq \infty$, is the space of functions on $\mathbb{R}^{3}$ such that

$$
\left\|u_{0}\right\|_{L_{\mathrm{uloc}}^{q}}=\sup _{x \in \mathbb{R}^{3}}\left\|u_{0}\right\|_{L^{q}(B(x, 1))}<\infty
$$

We also denote

$$
E^{q}=\mathrm{Cl}_{L_{\mathrm{uloc}}^{q}}\left(C_{0}^{\infty}\left(\mathbb{R}^{3}\right)\right)
$$


the closure of $C_{0}^{\infty}\left(\mathbb{R}^{3}\right)$ in the $L_{\text {uloc }}^{q}$-norm. We do not define solutions exactly as in [21] but instead recall a definition from [2]. For a cube $Q$ in $\mathbb{R}^{3}$, we denote by $Q^{*}$ and $Q^{* *}$ concentric cubes with side-lengths $4|Q|^{1 / 3} / 3$, and $5|Q|^{1 / 3} / 3$, respectively. Thus $Q \subset Q^{*} \subset Q^{* *}$. This is a slightly different definition from [2].

Definition 1.1 (Local energy solutions). A vector field $u \in L_{\text {loc }}^{2}\left(\mathbb{R}^{3} \times[0, T)\right)$, where $0<$ $T<\infty$, is a local energy solution to (1.1) with divergence-free initial data $u_{0} \in L_{\mathrm{loc}}^{2}\left(\mathbb{R}^{3}\right)$ if the following conditions hold:

1. $u \in \bigcap_{R>0} L^{\infty}\left(0, T ; L^{2}\left(B_{R}(0)\right)\right)$ and $\nabla u \in L_{\mathrm{loc}}^{2}\left(\mathbb{R}^{3} \times[0, T]\right)$,

2. for some $p \in L_{\mathrm{loc}}^{3 / 2}\left(\mathbb{R}^{3} \times(0, T)\right)$, the pair $(u, p)$ is a distributional solution to (1.1),

3. for all compact subsets $K$ of $\mathbb{R}^{3}$ we have $u(t) \rightarrow u_{0}$ in $L^{2}(K)$ as $t \rightarrow 0^{+}$,

4. $u$ is suitable in the sense of Caffarelli-Kohn-Nirenberg, i.e., for all non-negative $\phi \in$ $C_{0}^{\infty}\left(\mathbb{R}^{3} \times(0, T)\right)$, we have the local energy inequality

$$
2 \iint|\nabla u|^{2} \phi d x d t \leq \iint|u|^{2}\left(\partial_{t} \phi+\Delta \phi\right) d x d t+\iint\left(|u|^{2}+2 p\right)(u \cdot \nabla \phi) d x d t
$$

5. the function $t \mapsto \int u(x, t) \cdot w(x) d x$ is continuous on $[0, T)$ for any compactly supported $w \in L^{2}\left(\mathbb{R}^{3}\right)$,

6. for every cube $Q \subset \mathbb{R}^{3}$, there exists $p_{Q}(t) \in L^{3 / 2}(0, T)$ such that for $x \in Q^{*}$ and $0<t<T$,

$$
\begin{aligned}
p(x, t)-p_{Q}(t)= & -\frac{1}{3}|u(x)|^{2}+\text { p.v. } \int_{y \in Q^{* *}} K_{i j}(x-y)\left(u_{i}(y, s) u_{j}(y, s)\right) d y \\
& +\int_{y \notin Q^{* *}}\left(K_{i j}(x-y)-K_{i j}\left(x_{Q}-y\right)\right)\left(u_{i}(y, s) u_{j}(y, s) d y\right.
\end{aligned}
$$

where $x_{Q}$ is the center of $Q$ and $K_{i j}(y)=\partial_{i} \partial_{j}(4 \pi|y|)^{-1}$.

We say that $u$ is a local energy solution on $\mathbb{R}^{3} \times[0, \infty)$ if it is a local energy solution on $\mathbb{R}^{3} \times[0, T)$ for all $T<\infty$.

In comparison with the definitions in $[21,16,28,14,5]$, we do not require $u_{0} \in L_{\text {uloc }}^{2}$ or

$$
\operatorname{ess} \sup \sup _{0 \leq t<R^{2}} \int_{x_{0} \in \mathbb{R}^{3}} \int_{B_{R}\left(x_{0}\right)}|u(x, t)|^{2} d x+\sup _{x_{0} \in \mathbb{R}^{3}} \int_{0}^{R^{2}} \int_{B_{R}\left(x_{0}\right)}|\nabla u(x, t)|^{2} d x d t<\infty,
$$

for any $R>0$. For other low-regularity solution classes cf. [8, 18]. Suitability is an important property as it allows the application of the Caffarelli-Kohn-Nirenberg [7] and related regularity criteria and can be used to establish certain turbulent dynamics [9].

Local energy solutions are known to exist locally in time for initial data in $L_{\text {uloc }}^{2}(\Omega)$ where $\Omega$ is $\mathbb{R}^{3}[21,23,16,19]$ or $\mathbb{R}_{+}^{3}$ [26]. The global existence usually requires some type of decay or a structural assumption on the data $[21,16,19,4,8,5]$. In particular, in [5] two of the authors constructed global solutions for initial data satisfying

$$
\lim _{R \rightarrow \infty} \sup _{x_{0} \in \mathbb{R}^{3}} \frac{1}{R^{2}} \int_{B_{R}\left(x_{0}\right)}\left|u_{0}\right|^{2} d x=0 .
$$


In two dimensions, Basson constructed local solutions for a new class of (specified below) and global solutions for non-decaying data in the uniform space $L_{\text {uloc }}^{2}$ [1].

Recently, several existence results have appeared which allow for initial data in $L_{\text {loc }}^{2} \backslash$ $L_{\text {uloc }}^{2}$. In [2], two of the authors constructed local in time solutions for initial data in the space $\dot{M}_{\mathcal{C}}^{2,2}$ defined below. This construction is related to the idea of Basson [1], but is given in three dimensions as opposed to two, in a different setting, and uses a different approach to the pressure and estimates. In [10], Fernández-Dalgo and Lemarié-Rieusset constructed global solutions assuming the initial data $u_{0}$ satisfies

$$
\int_{\mathbb{R}^{3}} \frac{\left|u_{0}(x)\right|^{2}}{(1+|x|)^{2}} d x<\infty
$$

Fernández-Dalgo and Lemarié-Rieusset also included a new construction of discretely selfsimilar (DSS) solutions in [10] for any DSS data in $L_{\text {loc }}^{2}$ based on their global existence result (this gives a new proof of results in $[8,4]$; cf. also $[13,3]$ ) and is therefore of interest beyond the existence problem.

The purpose of this paper is to construct global solutions for the class of initial data $\stackrel{\circ}{\mathcal{C}}_{\mathcal{C}}^{2,2}$ introduced in [2], which strictly includes the initial data from $[5,10]$. For $n \in \mathbb{N}_{0}$, let

$$
Q_{n}=\left\{x:\left|x_{i}\right|<2^{n+1} \text { for } i=1,2,3\right\} .
$$

Denote

$$
S_{0}=\overline{Q_{0}}, \quad S_{n}=\overline{Q_{n} \backslash Q_{n-1}} \quad \text { for } n \in \mathbb{N} .
$$

Partition $S_{0}$ into 64 cubes of side-length 1 and $S_{n}$ into 56 cubes of side-length $2^{n}$. Let $\mathcal{C}$ be the collection of these cubes. Note that the number of cubes in $\bigcup_{i=0}^{n-1} S_{i}$ grows linearly in $n$.

The main features of the collection $\mathcal{C}$ are the following:

(i) The side-length of a cube is proportional to the distance of its center from the origin.

(ii) Adjacent cubes have comparable volumes.

(iii) If $\left|Q^{\prime}\right|<|Q|$, then the distance between the centers of $Q$ and $Q^{\prime}$ is proportional to $|Q|^{1 / 3}$.

(iv) The number of cubes $Q^{\prime}$ satisfying $\left|Q^{\prime}\right|<|Q|$ is bounded above by a constant multiple of $\log |Q|$.

For convenience we also refer to the collection of cubes in $\mathcal{C}$ contained in $S_{n}$ as $S_{n}$ and accordingly write $Q \in S_{n}$ if $Q$ is part of the collection $S_{n}$.

Our initial data space is an analogue of $L_{\text {uloc }}^{2}$ but adapted to the cover $\mathcal{C}$ and weighted.

Definition 1.2. Let $p \in[1, \infty)$ and $q \geq 0$. We have $f \in M_{\mathcal{C}}^{p, q}$ if

$$
\|f\|_{M_{\mathcal{C}}^{p, q}}^{p}:=\sup _{Q \in \mathcal{C}} \frac{1}{|Q|^{q / 3}} \int_{Q}|f(x)|^{p} d x<\infty .
$$

Let $\stackrel{\circ}{\mathcal{C}}^{p, q}$ be the set of $f \in M_{\mathcal{C}}^{p, q}$ such that

$$
\frac{1}{|Q|^{q / 3}} \int_{Q}|f|^{p} d x \rightarrow 0 \text { as }|Q| \rightarrow \infty, Q \in \mathcal{C} .
$$


Our main result asserts the existence of a global in time local energy solutions to the Navier-Stokes equations with data in $\stackrel{\circ}{\mathcal{C}}_{\mathcal{C}}^{2,2}$.

Theorem 1.3 (Global existence). Assume $u_{0} \in \stackrel{\circ}{M}_{\mathcal{C}}^{2,2}$ is divergence-free. Then there exists $u: \mathbb{R}^{3} \times(0, \infty) \rightarrow \mathbb{R}^{3}$ and $p: \mathbb{R}^{3} \times(0, \infty) \rightarrow \mathbb{R}$ so that $(u, p)$ is a local energy solution to the Navier-Stokes equations on $\mathbb{R}^{3} \times(0, \infty)$.

Theorem 1.3 is proven by first establishing the equivalence of the norm in $\stackrel{\circ}{\mathcal{C}}^{2, q}$ with the norm on the space ${\stackrel{\circ}{\mathcal{C}_{n}}}^{2, q}$ described next. Let

$$
\left\|u_{0}\right\|_{M_{\mathcal{C}_{n}}^{2, q}}^{2}=\sup _{Q \in \mathcal{C}_{n}} \frac{1}{|Q|^{q / 3}} \int_{Q}\left|u_{0}\right|^{2} d x
$$

where

$$
\mathcal{C}_{n}=\left\{Q_{n-1}\right\} \cup\left\{Q \in \mathcal{C}: \exists k \geq n \text { such that } Q \in S_{k}\right\},
$$

and, by (1.3), $Q_{n}=\bigcup_{i \leq n ; Q^{\prime} \in S_{i}} Q^{\prime}$. Thus we have replaced cubes in $\mathcal{C}$ with the side-lengths less than $2^{n}$ by a single cube of side-length $2^{n+1}$. We say $u_{0} \in M_{\mathcal{C}_{n}}^{2, q}$ if $\left\|u_{0}\right\|_{M_{\mathcal{C}_{n}}^{2, q}}<\infty$. The relationship between $\stackrel{\circ}{\mathcal{C}}^{2,2}$ and ${\stackrel{\circ}{\mathcal{C}_{n}}}^{2,2}$ is described in Lemma 2.1 below. The solution constructed in the proof of Theorem 1.3 also satisfies

$$
\underset{0<s<t}{\operatorname{ess~sup}}\|u(s)\|_{M_{\mathcal{C}_{n}}^{2,2}}^{2}+\sup _{Q \in \mathcal{C}_{n}} \frac{1}{|Q|^{2 / 3}} \int_{0}^{t} \int_{Q}|\nabla u(x, s)|^{2} d x d s \leq C\left\|u_{0}\right\|_{M_{\mathcal{C}_{n}}^{2,2}}^{2}
$$

for $t \leq T_{n}$, where $T_{n}$ is given in (3.4) below.

Note that Theorem 1.3 improves the constructions in $[2,10,5]$. The details are given in Section 2.

In [1], Basson considered local existence in two dimensions for initial data $u_{0}$ satisfying

$$
\sup _{R \geq 1} \frac{1}{R^{2}} \int_{|x| \leq R}\left|u_{0}\right|^{2} d x<\infty
$$

Basson left open the global existence of weak solutions in both $2 \mathrm{D}$ and $3 \mathrm{D}$. In three dimensions

$$
u_{0} \in \stackrel{\circ}{M}_{\mathcal{C}}^{2,2} \Longleftrightarrow \lim _{R \rightarrow \infty} \frac{1}{R^{2}} \int_{|x| \leq R}\left|u_{0}\right|^{2} d x=0 .
$$

Based on the global existence result Theorem 1.3, we expect that, in two dimensions, we get the global existence if

$$
\lim _{R \rightarrow \infty} \frac{1}{R^{2}} \int_{|x| \leq R}\left|u_{0}\right|^{2} d x=0,
$$

which is a subset of Basson's class that has very mild decay at spatial infinity.

Using the new a priori bounds in the proof of Theorem 1.3 allows us to establish the following eventual regularity result.

Theorem 1.4 (Eventual Regularity). Assume $u_{0} \in \stackrel{\circ}{M}_{\mathcal{C}}^{2,1}$ is divergence-free, and let $(u, p)$ be a local energy solution on $\mathbb{R}^{3} \times(0, \infty)$ with initial data $u_{0}$. Assume additionally that

$$
\underset{0<s<t}{\operatorname{ess} \sup }\|u(s)\|_{M_{\mathcal{C}}^{2,1}}^{2}+\sup _{Q \in \mathcal{C}} \frac{1}{|Q|^{1 / 3}} \int_{0}^{t} \int_{Q}|\nabla u(x, s)|^{2} d x d s<\infty,
$$

for all $t<\infty$. Then for any $\delta>0$, there exists a time $\tau$ depending only on $u_{0}$ and $\delta$ so that $u$ is smooth on

$$
\left\{(x, t): t>\max \left\{\delta|x|^{2}, \tau\right\}\right\}
$$


This follows from a priori bounds below, ideas in [5], and a partial regularity result of [7] (in particular, the version in [25]); cf. also [17,20]. This is variant of the usual notion of eventual regularity as it is not uniform in $x \in \mathbb{R}^{3}$. The class $\stackrel{\circ}{\mathcal{C}}^{2,1}$ excludes DSS data and leaves DSS solutions as the borderline candidate for the failure of eventual regularity even for our non-uniform version. Note that the shape of the regular set is consistent with that obtained for DSS solutions in [15]. In contrast, all self-similar solutions in this class are regular by [12].

It turns out that the spaces $M_{\mathcal{C}}^{p, q}$ are equivalent to certain Herz spaces. Let $A_{k}=\{x \in$ $\left.\mathbb{R}^{n}: 2^{k-1} \leq|x|<2^{k}\right\}$. For $n \in \mathbb{N}, s \in \mathbb{R}$ and $p, q \in(0, \infty]$, the homogeneous Herz space $\dot{K}_{p, q}^{s}\left(\mathbb{R}^{n}\right)$ is the space of functions $f \in L_{\text {loc }}^{p}\left(\mathbb{R}^{n} \backslash\{0\}\right)$ with finite norm

$$
\|f\|_{\dot{K}_{p, q}^{s}}= \begin{cases}\left(\sum_{k \in \mathbb{Z}} 2^{k s q}\|f\|_{L^{p}\left(A_{k}\right)}^{q}\right)^{1 / q} & \text { if } q<\infty \\ \sup _{k \in \mathbb{Z}} 2^{k s}\|f\|_{L^{p}\left(A_{k}\right)} & \text { if } q=\infty .\end{cases}
$$

The non-homogeneous Herz space $K_{p, q}^{s}\left(\mathbb{R}^{n}\right)$ is, with $A_{0}$ redefined as $B_{1}$,

$$
\|f\|_{K_{p, q}^{s}}= \begin{cases}\left(\sum_{k \in \mathbb{N}_{0}} 2^{k s q}\|f\|_{L^{p}\left(A_{k}\right)}^{q}\right)^{1 / q} & \text { if } q<\infty, \\ \sup _{k \in \mathbb{N}_{0}} 2^{k s}\|f\|_{L^{p}\left(A_{k}\right)} & \text { if } q=\infty .\end{cases}
$$

Then the space $M_{\mathcal{C}}^{p, q}$ is equivalent to the space with the norm

$$
\left(\int_{|x|<1}|f|^{p} d x\right)^{1 / p}+\sup _{R=2^{k}, k \in \mathbb{N}}\left(\frac{1}{R^{q}} \int_{R<|x|<2 R}|f|^{p} d x\right)^{1 / p},
$$

i.e., with the non-homogeneous Herz space $K_{p, \infty}^{-q}$. Local in time existence of mild solutions for large data in some subcritical weak Herz spaces (which include the Herz spaces) has been established by Tsutsui [31] ( $p>3$ is required). These spaces also appear in [15]

The paper is organized as follows. In Section 2 we reformulate the assumption $u_{0} \in \stackrel{M}{\mathcal{C}}_{\mathcal{C}}^{2,2}$ in a more usable context. In Section 3 we establish a new a priori bound that can be pushed to arbitrarily large times. In Section 4 we use the a priori bound from Section 3 to construct global solutions, while in Section 5 we prove Theorem 1.3.

\section{The space of initial data}

We first slightly reframe the assumption on the initial data.

Lemma 2.1. Let $f \in L_{\text {loc }}^{2}$. The following statements are equivalent
1. $f \in \stackrel{\circ}{\mathcal{C}}^{2,2}$,
2. $\|f\|_{M_{\mathcal{C}_{n}}^{2,2}} \rightarrow 0$ as $n \rightarrow \infty$,
3. $\lim _{R \rightarrow \infty} \frac{1}{R^{2}} \int_{B_{R}(0)}|f|^{2} \rightarrow 0$. 
Proof. We first check that our decay condition (2) matches (3). Assume $\|f\|_{M_{\mathcal{C}_{n}}^{2,2}} \rightarrow 0$ as $n \rightarrow$ $\infty$. This clearly implies $R^{-2} \int_{B_{R}(0)}|f|^{2} d x \rightarrow 0$. On the other hand, if $R^{-2} \int_{B_{R}(0)}^{c_{n}}|f|^{2} d x \rightarrow$ 0 , then, for $Q \in \mathcal{C}$ with $|Q|$ sufficiently large, we can make sure that

$$
\frac{1}{|Q|^{2 / 3}} \int_{Q}|f|^{2} \leq C \frac{1}{R^{2}} \int_{B_{R}(0)}|f|^{2} d x
$$

is arbitrarily small. This also implies

$$
\frac{1}{\left|Q_{n-1}\right|^{2 / 3}} \int_{Q_{n-1}}|f|^{2} d x \rightarrow 0
$$

as $n \rightarrow \infty$. Since $\mathcal{C}_{n}$ contains cubes with side-length larger than $2^{n}$ and $Q_{n-1}$, we may take $n$ sufficiently large and ensure that $\|f\|_{M_{\mathcal{C}_{n}}^{2,2}}$ is small. Thus (2) and (3) are equivalent.

Finally we check the equivalence of (1) and (2). Assume $f \in \stackrel{\circ}{\mathcal{C}}^{2,2}$. It suffices to show

$$
\frac{1}{2^{2 n}} \int_{Q_{n}}|f|^{2} d x \rightarrow 0
$$

Let $\epsilon>0$ be given. Then, since $f \in \stackrel{\circ}{M}_{\mathcal{C}}^{2,2}$, for any constant $\bar{C}$ there exists $N$ so that

$$
\frac{1}{|Q|^{2 / 3}} \int_{Q}|f|^{2} d x \leq \frac{\epsilon}{2 \bar{C}}
$$

provided $|Q| \geq 2^{3 N}$ and $Q \in \mathcal{C}$. We now have

$$
\frac{1}{2^{2 n}} \int_{Q_{n}}|f|^{2} d x \leq \sum_{Q \in \mathcal{C} ; Q \subset Q_{n} ;|Q| \geq 2^{3 N}} \frac{1}{2^{2 n}} \int_{Q}|f|^{2} d x+\sum_{Q \in \mathcal{C} ; Q \subset Q_{n} ;|Q|<2^{3 N}} \frac{1}{2^{2 n}} \int_{Q}|f|^{2} d x .
$$

By our choice of $N$ we have

$$
\sum_{Q \in \mathcal{C} ; Q \subset Q_{n} ;|Q| \geq 2^{3 N}} \frac{1}{2^{2 n}} \int_{Q}|f|^{2} d x \leq \frac{\epsilon}{2 \bar{C}} \sum_{Q \in \mathcal{C} ; Q \subset Q_{n}} \frac{|Q|^{2 / 3}}{2^{2 n}} \leq \frac{\epsilon}{2},
$$

where we have set $\bar{C}$ to be the uniform (in $n$ ) bound on the partial sum appearing above. On the other hand,

$$
\sum_{Q \in \mathcal{C} ; Q \subset Q_{n} ;|Q|<2^{3 N}} \frac{1}{2^{2 n}} \int_{Q}|f|^{2} d x \leq \frac{1}{2^{2 n}}\|f\|_{M_{\mathcal{C}}^{2,2}}^{2} \sum_{Q \in \mathcal{C} ;|Q|<2^{3 N}}|Q|^{2 / 3} .
$$

The last sum is bounded by $C 2^{2 N}$. By requiring $n$ to be large in comparison to $N$, we can ensure the last expression is smaller than $\epsilon / 2$. Hence, for a sufficiently large $n$,

$$
\frac{1}{2^{2 n}} \int_{Q_{n}}|f|^{2} d x<\epsilon
$$

and we have established that (1) implies (2).

Conversely, if $\|f\|_{\mathcal{C}_{n}} \rightarrow 0$, then $f \in \stackrel{\circ}{\mathcal{C}}^{2,2}$ follows immediately. 


\section{A priori bounds}

In this section we work exclusively with $\mathcal{C}_{n}$, where $n \in \mathbb{N}$ is given. Let $Q_{n}=\bigcup_{m \leq n ; Q^{\prime} \in S_{m}} Q^{\prime}$.

\subsection{Pressure estimate}

We adapt the pressure estimates in [2] to the $M_{\mathcal{C}_{n}}^{2, q}$ framework. Denote

$$
G_{i j} f(x)=R_{i} R_{j} f(x)=-\frac{1}{3} \delta_{i j} f(x)+\text { p.v. } \int K_{i j}(x-y) f(y) d y,
$$

where $R_{i}$ denotes the $i$-th Riesz transform and

$$
K_{i j}(y)=\partial_{i} \partial_{j} \frac{1}{4 \pi|y|}=\frac{-\delta_{i j}|y|^{2}+3 y_{i} y_{j}}{4 \pi|y|^{5}} .
$$

Fix a cube $Q \subset \mathbb{R}^{3}$, and let $Q^{*}, Q^{* *}$ be as defined preceding Definition 1.1. For $x \in Q^{*}$, let

$$
\begin{aligned}
G_{i j}^{Q} f(x)= & -\frac{1}{3} \delta_{i j} f(x)+\text { p.v. } \int_{y \in Q^{* *}} K_{i j}(x-y) f(y) d y \\
& +\int_{y \notin Q^{* *}}\left(K_{i j}(x-y)-K_{i j}\left(x_{Q}-y\right)\right) f(y) d y .
\end{aligned}
$$

We say that a pressure $p$, associated to a local energy solution $u$, satisfies the pressure expansion if

$$
p(x, t)-p_{Q}(t)=\left(G_{i j}^{Q} u_{i} u_{j}\right)(x, t), \quad x \in Q^{*},
$$

for some $p_{Q}(t) \in L^{3 / 2}(0, T)$.

Lemma 3.1. Fix $n \in \mathbb{N}$. Assume $u$ is a local energy solution to (1.1) on $\mathbb{R}^{3} \times[0, T]$ with an associated pressure $p$. Then, for $Q \in \mathcal{C}_{n}$ and $t<T$,

$$
\begin{aligned}
& \frac{1}{|Q|^{1 / 3}} \int_{0}^{t} \int_{Q^{*}}\left|p-p_{Q}(s)\right|^{3 / 2} d x d s \\
& \quad \leq C \sup _{Q^{\prime} \in \mathcal{C}_{n} ; Q^{\prime} \cap Q^{* *} \neq \emptyset} \frac{1}{\left|Q^{\prime}\right|^{1 / 3}} \int_{0}^{t} \int_{Q^{\prime}}|u|^{3} d x d s \\
& \quad+C|Q|^{q / 2-5 / 6}\left(\log \left\langle|Q|^{1 / 3} / 2^{n}\right\rangle\right)^{3 / 2} \int_{0}^{t}\left(\sup _{Q^{\prime} \in \mathcal{C}_{n}} \frac{1}{\left|Q^{\prime}\right| q / 3} \int_{Q^{\prime}}|u(x, s)|^{2} d x\right)^{3 / 2} d s
\end{aligned}
$$

where we denote $\langle x\rangle=\left(1+|x|^{2}\right)^{1 / 2}$.

Proof. The proof follows [2, Proof of Lemma 2.1] with an improved bound on the far part of the pressure (cf. the last term in (3.2)). For $x \in Q^{*}$, write

$$
p(x, t)-p_{Q}(t)=I_{\text {near }}(x, t)+I_{\text {far }}(x, t),
$$

where $I_{\text {near }}(x, t)$ is the sum of the first two terms on the right hand side of $(3.1)$ and $I_{\text {far }}(x, t)$ is the last term where $f=u_{i} u_{j}$.

The Calderón-Zygmund inequality implies

$$
\frac{1}{|Q|^{1 / 3}} \int_{0}^{t} \int_{Q^{*}}\left|I_{\text {near }}\right|^{3 / 2} d x d s \leq \frac{C}{|Q|^{1 / 3}} \int_{0}^{t} \int_{Q^{* *}}|u|^{3} d x d s .
$$


Since $|Q| \sim\left|Q^{\prime}\right|$ whenever $Q^{\prime} \in \mathcal{C}_{n}$ and $Q^{\prime} \cap Q^{* *} \neq 0$ and there are a bounded number of cubes satisfying this property (independent of $Q$ and $n$ ), we have

$$
\frac{1}{|Q|^{1 / 3}} \int_{0}^{t} \int_{Q^{* *}}|u|^{3} d x d s \leq C \sup _{Q^{\prime} \cap Q^{* *} \neq \emptyset, Q^{\prime} \in \mathcal{C}_{n}} \frac{1}{\left|Q^{\prime}\right|^{1 / 3}} \int_{0}^{t} \int_{Q^{\prime}}|u|^{3} d x d s .
$$

We now estimate $I_{\text {far }}$. Recall that if $x_{Q}$ is the center of $Q$, then $Q^{*}$ is the cube centered at $x_{Q}$ with the side-length $4|Q|^{1 / 3} / 3$, and $Q^{* *}$ is the same but with the side-length $5|Q|^{1 / 3} / 3$. Also, if $Q \in \mathcal{C}_{n}$, then $Q^{*}$ and $Q^{* *}$ only overlap cubes in $\mathcal{C}_{n}$ which are adjacent to $Q$. If $y \notin Q^{* *}$ and $x \in Q^{*}$, then

$$
\left|K_{i j}(x-y)-K_{i j}\left(x_{Q}-y\right)\right| \leq \frac{C|Q|^{1 / 3}}{|x-y|^{4}} .
$$

Let $m \in \mathbb{N}$ be such that $|Q|=2^{3 m}$. For $x \in Q^{*}$, we have

$$
\begin{aligned}
\left|I_{\mathrm{far}}(x, t)\right| \leq & C|Q|^{1 / 3} \int_{\mathbb{R}^{3} \backslash Q^{* *}} \frac{1}{|x-y|^{4}}|u(y, t)|^{2} d y \\
\leq & C \sum_{Q^{\prime} \in \mathcal{S}_{1}}|Q|^{1 / 3} \int_{Q^{\prime} \cap\left(Q^{* *}\right)^{c}} \frac{1}{|x-y|^{4}}|u|^{2} d y \\
& +C \sum_{Q^{\prime} \in \mathcal{S}_{2}}|Q|^{1 / 3} \int_{Q^{\prime} \cap\left(Q^{* *}\right)^{c}} \frac{1}{|x-y|^{4}}|u|^{2} d y
\end{aligned}
$$

where $\mathcal{S}_{1}$ is the collection of $Q^{\prime} \in \mathcal{C}_{n}$ such that $Q^{\prime} \subset Q_{m+1}$ while $\mathcal{S}_{2}$ is the collection of $Q^{\prime} \in \mathcal{C}_{n}$ such that $Q^{\prime} \subset Q_{m+1}^{c}$.

We first focus on $\mathcal{S}_{1}$. The number of cubes $Q^{\prime}$ in $\mathcal{S}_{1}$ is bounded above by $C \log \left\langle|Q|^{1 / 3} / 2^{n}\right\rangle$. Furthermore, $|Q|^{1 / 3} \sim\left|x_{Q}-x_{Q^{\prime}}\right|$ whenever $Q^{\prime} \in \mathcal{S}_{1}$. We claim that if $y \in Q^{\prime} \cap\left(Q^{* *}\right)^{c}$ and $x \in Q$, then $|x-y| \sim\left|x_{Q}-x_{Q}^{\prime}\right|$. This is clear if $Q^{\prime}$ and $Q$ are not adjacent. If they are adjacent, then we obviously have $\left|x_{Q}-x_{Q^{\prime}}\right| \sim 2^{m}$ and $|x-y| \leq 3 \cdot 2^{m}$. For the lower bound, since $y \notin Q^{* *}$, we must have $|x-y| \geq 2^{m-1}$. This proves our claim. Hence,

$$
\begin{aligned}
& \sum_{Q^{\prime} \in \mathcal{S}_{1}}|Q|^{1 / 3} \int_{Q^{\prime} \cap\left(Q^{* *}\right)^{c}} \frac{1}{|x-y|^{4}}|u|^{2} d y \leq C \sum_{Q^{\prime} \in \mathcal{S}_{1}} \frac{|Q|^{1 / 3}}{|Q|^{4 / 3}} \int_{Q^{\prime}}|u|^{2} d y \\
& \leq C \sup _{Q^{\prime} \in \mathcal{S}_{1}} \frac{\log \left\langle|Q|^{1 / 3} / 2^{n}\right\rangle}{|Q|} \int_{Q^{\prime}}|u|^{2} d y \\
& \quad=C \sup _{Q^{\prime} \in \mathcal{S}_{1}} \frac{\left|Q^{\prime}\right|^{q / 3} \log \left\langle|Q|^{1 / 3} / 2^{n}\right\rangle}{|Q|} \frac{1}{\left|Q^{\prime}\right|^{q / 3}} \int_{Q^{\prime}}|u|^{2} d y \\
& \leq C|Q|^{q / 3-1} \log \left\langle|Q|^{1 / 3} / 2^{n}\right\rangle \sup _{Q^{\prime} \in \mathcal{C}_{n}} \frac{1}{\left|Q^{\prime}\right|^{q / 3}} \int_{Q^{\prime}}|u|^{2} d x .
\end{aligned}
$$

On the other hand, if $Q^{\prime} \in \mathcal{S}_{2}$ then $Q^{\prime} \cap\left(Q^{* *}\right)^{c}=Q^{\prime}$ (because $Q^{* *}$ only overlaps with cubes adjacent to $Q$ which are all subsets of $\left.Q_{m+1}\right)$. Hence, the $\mathcal{S}_{2}$ term is bounded as

$$
\begin{aligned}
& \sum_{Q^{\prime} \in \mathcal{S}_{2}}|Q|^{1 / 3} \int_{Q^{\prime}} \frac{1}{|x-y|^{4}}|u|^{2} d y=\sum_{l \geq m+1} \sum_{Q^{\prime} \in S_{l} \cap \mathcal{S}_{2}} \frac{|Q|^{1 / 3}}{\left|x_{Q}-x_{Q^{\prime}}\right|^{4}} \int_{Q^{\prime}}|u|^{2} d x \\
& \leq C|Q|^{1 / 3} \sum_{l \geq m+1} \frac{1}{2^{(4-q) l}} \sup _{Q^{\prime} \in \mathcal{C}_{n}} \frac{1}{\left|Q^{\prime}\right| q / 3} \int_{Q^{\prime}}|u|^{2} d x \leq \frac{C|Q|^{1 / 3}}{2^{(4-q) m}} \sup _{Q^{\prime} \in \mathcal{C}_{n}} \frac{1}{\left|Q^{\prime}\right| q / 3} \int_{Q^{\prime}}|u|^{2} d x \\
& \leq C|Q|^{q / 3-1} \sup _{Q^{\prime} \in \mathcal{C}_{n}} \frac{1}{\left|Q^{\prime}\right|^{q / 3}} \int_{Q^{\prime}}|u|^{2} d x .
\end{aligned}
$$


Combining these estimates yields (3.3), and the proof is concluded.

\subsection{Main estimate}

In this section we obtain an estimate for solutions in the spaces $M_{\mathcal{C}_{n}}^{2, q}$.

Our estimate for local cubic terms is the following.

Lemma 3.2. Let $u: \mathbb{R}^{3} \times(0, T) \rightarrow \mathbb{R}^{3}$. Then, given $\varepsilon>0$, we have

$$
\begin{aligned}
\frac{1}{|Q|^{1 / 3}} \int_{0}^{t} \int_{Q}|u|^{3} d x d s \leq & C(\varepsilon)|Q|^{q-4 / 3} \int_{0}^{t}\left(\frac{1}{|Q|^{q / 3}} \int_{Q}|u|^{2} d x\right)^{3} d s \\
& +\varepsilon \int_{0}^{t} \int_{Q}|\nabla u|^{2} d x d s \\
& +C|Q|^{q / 2-5 / 6} \int_{0}^{t}\left(\frac{1}{|Q|^{q / 3}} \int_{Q}|u|^{2} d x\right)^{3 / 2} d s
\end{aligned}
$$

for any cube $Q \subset \mathbb{R}^{3}$.

Proof. By the Gagliardo-Nirenberg and Hölder inequalities we have

$$
\begin{aligned}
\frac{1}{|Q|^{1 / 3}} & \int_{0}^{t} \int_{Q}|u|^{3} d x d s \\
\leq & C \frac{1}{|Q|^{1 / 3}} \int_{0}^{t}\left(\int_{Q}|u|^{2} d x\right)^{3 / 4}\left(\int_{Q}|\nabla u|^{2} d x\right)^{3 / 4} d s \\
& +C \frac{1}{|Q|^{1 / 3}} \int_{0}^{t} \frac{1}{|Q|^{1 / 2}}\left(\int_{Q}|u|^{2} d x\right)^{3 / 2} d s \\
\leq & C \frac{1}{|Q|^{1 / 3}}\left(\int_{0}^{t}\left(\int_{Q}|u|^{2} d x\right)^{3} d s\right)^{1 / 4}\left(\int_{0}^{t} \int_{Q}|\nabla u|^{2} d x d s\right)^{3 / 4} \\
& +C \frac{|Q|^{q / 2}}{|Q|^{5 / 6}} \int_{0}^{t}\left(\frac{1}{|Q|^{q / 3}} \int_{Q}|u|^{2} d x\right)^{3 / 2} d s .
\end{aligned}
$$

Using Young's inequality $a b \lesssim a^{4}+b^{4 / 3}$, the last expression is bounded by

$$
\begin{aligned}
\leq & C \frac{|Q|^{q / 4}}{|Q|^{1 / 3}}\left(\int_{0}^{t}\left(\frac{1}{|Q|^{q / 3}} \int_{Q}|u|^{2} d x\right)^{3} d s\right)^{1 / 4}\left(\int_{0}^{t} \int_{Q}|\nabla u|^{2} d x d s\right)^{3 / 4} \\
& +C|Q|^{q / 2-5 / 6} \int_{0}^{t}\left(\frac{1}{|Q|^{q / 3}} \int_{Q}|u|^{2} d x\right)^{3 / 2} d s \\
\leq & C(\varepsilon)|Q|^{q-4 / 3} \int_{0}^{t}\left(\frac{1}{|Q|^{q / 3}} \int_{Q}|u|^{2} d x\right)^{3} d s+\varepsilon \int_{0}^{t} \int_{Q}|\nabla u|^{2} d x d s \\
& +C|Q|^{q / 2-5 / 6} \int_{0}^{t}\left(\frac{1}{|Q|^{q / 3}} \int_{Q}|u|^{2} d x\right)^{3 / 2} d s,
\end{aligned}
$$

and the proof is concluded.

We study the local energy by way of cutoff functions $\phi_{Q}$ which we now define. Let $\phi$ be a radial smooth cutoff function such that $\phi=1$ in $[-1 / 2,1 / 2]^{3}$ and $\phi=0$ off of $[-2 / 3,2 / 3]^{3}$ 
with $\phi$ non-increasing in $|x|$. For $Q \in \mathcal{C}_{n}$, let $\phi_{Q}$ be the translation and dilation of $\phi$ so that $\phi_{Q}$ equals 1 on $Q$ and vanishes off of $Q^{*}$. Then, $\left\|\partial^{\lambda} \phi_{Q}(x)\right\|_{L^{\infty}} \leq C(\lambda) /|Q|^{|\lambda| / 3}$ where $C$ does not depend on $Q$ and $\lambda$ is any multi-index.

We need the following version of the Grönwall inequality.

Lemma 3.3. Suppose $f(t) \in L_{\mathrm{loc}}^{\infty}([0, T) ;[0, \infty))$ satisfies, for some $m \geq 1$,

$$
f(t) \leq a+\int_{0}^{t}\left(b_{1} f(s)+b_{2} f(s)^{m}\right) d s, \quad 0<t<T,
$$

where $a, b>0$, then for $T_{0}=\min \left(T, T_{1}\right)$, with

$$
T_{1}=\frac{a}{b_{1} 2 a+b_{2}(2 a)^{m}}
$$

we have $f(t) \leq 2 a$ for $t \in\left(0, T_{0}\right)$.

The proof is obtained by a barrier argument; cf. [5]) for details.

Denote

$$
\alpha_{n}(t)=\|u(t)\|_{M_{\mathcal{C}_{n}}^{2,2}}^{2}
$$

and

$$
\beta_{n}(t)=\sup _{Q \in \mathcal{C}_{n}} \frac{1}{|Q|^{2 / 3}} \int_{0}^{t} \int_{Q}|\nabla u(x, s)|^{2} d x d s .
$$

We now state the bound on the existence times. It is motivated by an estimate in [14]. Theorem 3.4. Assume $u_{0} \in M_{\mathcal{C}_{n}}^{2,2}$, is divergence-free, and let $(u, p)$ be a local energy solution with initial data $u_{0}$ on $\mathbb{R}^{3} \times(0, \infty)$. Assume additionally that

$$
\underset{0<s<t}{\operatorname{ess} \sup } \alpha_{n}(s)+\beta_{n}(t)<\infty,
$$

for all $t<\infty$. Then there exist constants $c_{0}$ and $c_{1}$ both independent of $n$ so that, setting

$$
T_{n}=\frac{c_{1}}{2^{-2 n}+\left\|u_{0}\right\|_{M_{\mathcal{C}_{n}}^{2,2}}^{4}}
$$

we have

$$
\sup _{0<s<T_{n}}\|u(s)\|_{M_{\mathcal{C}_{n}}^{2,2}}^{2}+\sup _{Q \in \mathcal{C}_{n}} \frac{1}{|Q|^{2 / 3}} \int_{0}^{T_{n}} \int_{Q}|\nabla u|^{2} d x d s \leq 2 c_{0}\left\|u_{0}\right\|_{M_{\mathcal{C}_{n}}^{2,2}}^{2} .
$$

Let us briefly explain why this bound is useful. In comparison to the analogous estimate in [2], we have control over the time scales via the parameter $n$. Indeed, if $\left\|u_{0}\right\|_{M_{\mathcal{C}_{n}}^{2,2}} \rightarrow 0$ as $n \rightarrow \infty$, then $T_{n} \rightarrow \infty$. This leads to a global solution in the next section.

Proof of Theorem 3.4. Fix $Q \in \mathcal{C}_{n}$. The local energy inequality and the item 5 of Definition 1.1 give

$$
\begin{aligned}
\frac{1}{2} \int|u(x, t)|^{2} \phi_{Q}(x) d x+\int_{0}^{t} \int|\nabla u(x, s)|^{2} \phi_{Q}(x) d x d s \\
\leq \frac{1}{2} \int|u(x, 0)|^{2} \phi_{Q}(x) d x+\frac{1}{2} \int_{0}^{t} \int|u(x, s)|^{2} \Delta \phi_{Q}(x) d x d s \\
\quad+\frac{1}{2} \int_{0}^{t} \int\left(|u(x, s)|^{2} u \cdot \nabla \phi_{Q}(x)+2\left(p(x, s)-p_{Q}(s)\right) u(x, s) \cdot \nabla \phi_{Q}(x)\right) d x d s .
\end{aligned}
$$


For the linear term we have,

$$
\begin{aligned}
& \frac{1}{2} \int_{0}^{t} \int|u|^{2} \Delta \phi_{Q} d x d s \leq \frac{C}{|Q|^{2 / 3}} \int_{0}^{t} \sup _{Q^{\prime} \cap Q^{*} \neq \emptyset} \int_{Q^{\prime}}|u|^{2} d x d s \\
& \quad \leq C \int_{0}^{t} \sup _{Q^{\prime} \cap Q^{*} \neq \emptyset} \frac{1}{\left|Q^{\prime}\right|^{q / 3}} \frac{\left|Q^{\prime}\right|^{q / 3}}{|Q|^{2 / 3}} \int_{Q^{\prime}}|u|^{2} d x d s \leq C|Q|^{(q-2) / 3} \int_{0}^{t} \alpha_{n}(s) d s,
\end{aligned}
$$

where we used the fact that $\left|Q^{\prime}\right|$ and $|Q|$ are comparable.

The remaining terms are bounded using Lemmas 3.1 and 3.2. This leads to

$$
\begin{aligned}
& \int|u(x, t)|^{2} \phi_{Q}(x) d x+2 \int_{0}^{t} \int|\nabla u(x, s)|^{2} \phi_{Q}(x) d x d s \\
& \leq \int|u(x, 0)|^{2} \phi_{Q}(x) d x+C|Q|^{(q-2) / 3} \int_{0}^{t} \alpha_{n}(s) d s \\
& \quad+C\left(\log \left\langle|Q|^{1 / 3} / 2^{n}\right\rangle\right)^{3 / 2}|Q|^{q / 2-5 / 6} \int_{0}^{t} \alpha_{n}(s)^{3 / 2} d s \\
& +C|Q|^{q-4 / 3} \int_{0}^{t} \alpha_{n}(s)^{3} d s \\
& +\varepsilon \sup _{Q^{\prime} \in \mathcal{C}_{n} ; Q^{\prime} \cap Q^{* *} \neq \emptyset}\left|Q^{\prime}\right|^{q / 2-1 / 3} \frac{1}{\left|Q^{\prime}\right|^{q / 3}} \int_{0}^{t} \int_{Q^{\prime}}|\nabla u|^{2} d x d s,
\end{aligned}
$$

where $\varepsilon>0$ is fixed (with the choice explained after (3.6) below); the constants between (3.5) and (3.6) depend on $\varepsilon$. Next, plug in $q=2$ and divide through by $|Q|^{2 / 3}$. Then, by Young's inequality,

$$
\begin{aligned}
& C\left(\log \left\langle|Q|^{1 / 3} / 2^{n}\right\rangle\right)^{3 / 2}|Q|^{q / 2-5 / 6-2 / 3} \int_{0}^{t} \alpha_{n}(s)^{3 / 2} d s \\
& \quad \leq C\left(\log \left\langle|Q|^{1 / 3} / 2^{n}\right\rangle\right)^{2}|Q|^{(1-5 / 6-2 / 3) 4 / 3} \int_{0}^{t} \alpha_{n}(s) d s+\int_{0}^{t} \alpha_{n}(s)^{3} d s \\
& \quad=C \frac{\left(\log \left\langle|Q|^{1 / 3} / 2^{n}\right\rangle\right)^{2}}{\left(|Q|^{1 / 3} / 2^{n}\right)^{2} 2^{2 n}} \int_{0}^{t} \alpha_{n}(s) d s+\int_{0}^{t} \alpha_{n}(s)^{3} d s .
\end{aligned}
$$

For $Q \in \mathcal{C}_{n}$

$$
\frac{\left(\log \left\langle|Q|^{1 / 3} / 2^{n}\right\rangle\right)^{2}}{\left(|Q|^{1 / 3} / 2^{n}\right)^{2}}
$$

is bounded and the bound is independent of $n$. Hence,

$$
\begin{gathered}
C\left(\log \left\langle|Q|^{1 / 3} / 2^{n}\right\rangle\right)^{3 / 2}|Q|^{q / 2-5 / 6-2 / 3} \int_{0}^{t} \alpha_{n}(s)^{3 / 2} d s \\
\leq C 2^{-2 n} \int_{0}^{t} \alpha_{n}(s) d s+\int_{0}^{t} \alpha_{n}(s)^{3} d s .
\end{gathered}
$$

Using this in (3.5) divided by $|Q|^{2 / 3}$ leads to

$$
\begin{aligned}
& \frac{1}{|Q|^{2 / 3}} \int|u(x, t)|^{2} \phi_{Q}(x) d x+\frac{2}{|Q|^{2 / 3}} \int_{0}^{t} \int|\nabla u(x, s)|^{2} \phi_{Q}(x) d x d s \\
& \leq \leq \frac{1}{|Q|^{2 / 3}} \int|u(x, 0)|^{2} \phi_{Q}(x) d x+\frac{C}{2^{2 n}} \int_{0}^{t} \alpha_{n}(s) d s+C \int_{0}^{t} \alpha_{n}(s)^{3} d s \\
& \quad+\sup _{Q^{\prime} \in \mathcal{C}_{n} ; Q^{\prime} \cap Q^{* *} \neq \emptyset} \frac{1}{\left|Q^{\prime}\right|^{2 / 3}} \int_{0}^{t} \int_{Q^{\prime}}|\nabla u|^{2} d x d s,
\end{aligned}
$$


where, to obtain the last line, $\varepsilon$ is chosen to be small enough so that $\varepsilon\left|Q^{\prime}\right|^{2 / 3} /|Q|^{2 / 3} \leq 1$ whenever $Q^{\prime} \cap Q^{* *} \neq \emptyset$. Such $\varepsilon$ can be chosen independently of $Q$ and $n$. Taking the supremum over $Q \in \mathcal{C}_{n}$ and absorbing the gradient term in the left hand side gives

$$
\|u(t)\|_{M_{\mathcal{C}_{n}}^{2,2}}^{2} \leq c_{0}\left\|u_{0}\right\|_{M_{\mathcal{C}_{n}}^{2,2}}^{2}+C 2^{-2 n} \int_{0}^{t}\|u(s)\|_{M_{\mathcal{C}_{n}}^{2,2}}^{2} d s+C \int_{0}^{t}\|u(s)\|_{M_{\mathcal{C}_{n}}^{2,2}}^{6} d s,
$$

where $c_{0}$ is a fixed constant depending only on $\phi$ chosen to ensure

$$
\sup _{Q \in \mathcal{C}_{n}} \frac{1}{|Q|^{2 / 3}} \int\left|u_{0}\right|^{2} \phi_{Q} d x \leq c_{0}\left\|u_{0}\right\|_{M_{\mathcal{C}_{n}}^{2,2}}^{2}
$$

By Lemma 3.3 we obtain

$$
\|u(t)\|_{M_{\mathcal{C}_{n}}^{2,2}}^{2} \leq 2 c_{0}\left\|u_{0}\right\|_{M_{\mathcal{C}_{n}}^{2,2}}^{2}
$$

provided

$$
t \leq \frac{C}{2^{-2 n}+\left\|u_{0}\right\|_{M_{\mathcal{C}_{n}}^{2,2}}^{4}} .
$$

Thus letting $c_{1}$ be the constant from the above inequality completes the proof.

Remark 3.5. Theorem 3.4 can be re-formulated for $M_{\mathcal{C}_{n}}^{2, q}$ for $0 \leq q<2$. To prove this, just start at (3.5) and proceed with a different choice of $q$. Doing so for $q=1$ leads to the estimate

$$
\sup _{0<s<\bar{T}_{n}}\|u(s)\|_{M_{\mathcal{C}_{n}}^{2,1}}^{2}+\sup _{Q \in \mathcal{C}_{n}} \frac{1}{|Q|^{1 / 3}} \int_{0}^{\bar{T}_{n}} \int_{Q}|\nabla u|^{2} d x d s \leq 2 c_{0}\left\|u_{0}\right\|_{M_{\mathcal{C}_{n}}^{2,1}}^{2}
$$

where

$$
\bar{T}_{n} \sim \frac{1}{2^{-2 n}+2^{-2 n}\left\|u_{0}\right\|_{M_{\mathcal{C}_{n}}^{2,1}}^{4}}
$$

If additionally $u_{0} \in \stackrel{\circ}{M}_{\mathcal{C}}^{2,1}$, then $\left\|u_{0}\right\|_{M_{\mathcal{C}_{n}}^{2,1}} \rightarrow 0$ as $n \rightarrow \infty$ and we may take $\bar{T}_{n} \sim 2^{2 n}$ for large $n$.

\section{Construction of global solutions}

Recall that the initial data can be approximated by the following lemma proven in [2]. The second conclusion is new and follows from observations in Section 2.

Lemma 4.1. Assume $f \in \stackrel{\circ}{\mathcal{C}}^{2,2}$ is divergence-free. For every $\epsilon>0$ there exists a divergencefree $g \in L^{2}$ such that $\|f-g\|_{M_{\mathcal{C}}^{2,2}} \leq \epsilon$ and $\|f-g\|_{M_{\mathcal{C}_{n}}^{2,2}}<\epsilon$ for every $n \in \mathbb{N}$ ( $g$ is independent of $n$ ).

Our proof follows the global existence argument in [5] very closely. The basic elements of this argument were first written down in [21] and later elaborated in [16]. See also $[22,19,23,26]$. 
Proof. We argue by induction. For $n \in \mathbb{N}$, let $u_{0}^{n}$ be as in Lemma 4.1 where $\left\|u_{0}-u_{0}^{n}\right\|_{M_{\mathcal{C}}^{2,2}}<$ $\frac{1}{n}$ and $\left\|u_{0}-u_{0}^{n}\right\|_{M_{\mathcal{C}_{k}}^{2,2}}<1 / n$ for all $k \in \mathbb{N}$. Let $u^{n}$ and $\tilde{p}^{n}$ be a global solution in the Leray class with $u^{n}(0)=u_{0}^{n}$. By the classical theory (see [30] for a reference) we may furthermore assume that $u^{n}$ is suitable and $\tilde{p}^{n}$ satisfies the local pressure expansion. Let $T_{n}=\inf _{j \geq n} \bar{T}_{j}$ where $\bar{T}_{j}$ is the time-scale from Theorem 3.4. This sequence is non-decreasing and $T_{n} \rightarrow \infty$ as $n \rightarrow \infty$. Let $B_{n}$ denote the ball centered at the origin of radius $n$. Then, Theorem 3.4 implies that $u^{n}$ are uniformly bounded in the class from inequalities [16, (4.1)-(4.4)] on $B_{1} \times\left[0, T_{1}\right]$. Hence, there exists a sub-sequence $u^{1, k}$ that converges to a vector field $u_{1}$ on $B_{1} \times\left(0, T_{1}\right)$ in the following sense

$$
\begin{array}{ll}
u^{1, k} \stackrel{*}{\rightarrow} u_{1} & \text { in } L^{\infty}\left(0, T_{1} ; L^{2}\left(B_{1}\right)\right) \\
u^{1, k} \rightarrow u_{1} & \text { in } L^{2}\left(0, T_{1} ; H^{1}\left(B_{1}\right)\right) \\
u^{1, k} \rightarrow u_{1} & \text { in } L^{3}\left(0, T_{1} ; L^{3}\left(B_{1}\right)\right) .
\end{array}
$$

By Theorem 3.4, all $u^{1, k}$ are also uniformly bounded on $B_{n} \times\left[0, T_{n}\right]$ for $n \in \mathbb{N}, n \geq$ 2. Therefore, we can inductively extract subsequences $\left\{u^{n, k}\right\}_{k \in \mathbb{N}}$ from $\left\{u^{n-1, k}\right\}_{k \in \mathbb{N}}$ which converge to a vector field $u_{n}$ on $B_{n} \times\left(0, T_{n}\right)$ as $k \rightarrow \infty$ in the following sense

$$
\begin{array}{ll}
u^{n, k} \stackrel{*}{\rightarrow} u_{n} & \text { in } L^{\infty}\left(0, T_{n} ; L^{2}\left(B_{n}\right)\right) \\
u^{n, k} \rightarrow u_{n} & \text { in } L^{2}\left(0, T_{n} ; H^{1}\left(B_{n}\right)\right) \\
u^{n, k} \rightarrow u_{n} & \text { in } L^{3}\left(0, T_{n} ; L^{3}\left(B_{n}\right)\right) .
\end{array}
$$

Let $\tilde{u}_{n}$ be the extension by 0 of $u_{n}$ to $\mathbb{R}^{3} \times(0, \infty)$. Note that, at each step, $\tilde{u}_{n}$ agrees with $\tilde{u}_{n-1}$ on $B_{n-1} \times\left(0, T_{n-1}\right)$. Let $u=\lim _{n \rightarrow \infty} \tilde{u}_{n}$. Then, $u=u_{n}$ on $B_{n} \times\left(0, T_{n}\right)$ for every $n \in \mathbb{N}$.

Let $u^{(k)}=u^{k, k}$ on $B_{k} \times\left(0, T_{k}\right)$ and equal 0 elsewhere. Then, for every fixed $n$ and as $k \rightarrow \infty$,

$$
\begin{array}{ll}
u^{(k)} \stackrel{*}{\rightarrow} u & \text { in } L^{\infty}\left(0, T_{n} ; L^{2}\left(B_{n}\right)\right) \\
u^{(k)} \rightarrow u & \text { in } L^{2}\left(0, T_{n} ; H^{1}\left(B_{n}\right)\right) \\
u^{(k)} \rightarrow u & \text { in } L^{3}\left(0, T_{n} ; L^{3}\left(B_{n}\right)\right) .
\end{array}
$$

Based on the uniform bounds for the approximates, we have that $u$ satisfies

$$
\begin{aligned}
& \sup _{0<t \leq T_{n}} \sup _{x_{0} \in \mathbb{R}^{3}} \int_{B_{n}\left(x_{0}\right)}|u(x, t)|^{2} d x \\
& \quad+\sup _{x_{0} \in \mathbb{R}^{3}} \int_{0}^{T_{n}} \int_{B_{n}\left(x_{0}\right)}|\nabla u(x, t)|^{2} d x d s \leq C \sup _{x_{0} \in \mathbb{R}^{3}} \int_{B_{n}\left(x_{0}\right)}\left|u_{0}\right|^{2} d x .
\end{aligned}
$$

The pressure is dealt with as in $[19, \S 3]$. Let

$$
\begin{aligned}
p^{(k)}(x, t)= & -\frac{1}{3}\left|u^{(k)}\right|^{2}(x, t)+\text { p.v. } \int_{B_{2}} K_{i j}(x-y)\left(u_{i}^{(k)} u_{j}^{(k)}\right)(y, t) d y \\
& + \text { p.v. } \int_{B_{2}^{c}}\left(K_{i j}(x-y)-K_{i j}(-y)\right)\left(u_{i}^{(k)} u_{j}^{(k)}\right)(y, t) d y,
\end{aligned}
$$

which differs from the pressure associated to $u^{(k)}$ by a function of $t$ which is constant in $x$, and so $u^{(k)}$ with the above pressure $p^{(k)}$ is also a distributional solution to (1.1). 
Since $u^{(k)}$ converges to $u$ in the above sense, it follows that $p^{(k)} \rightarrow p$ in $L^{3 / 2}\left(0, T_{m} ; L^{3 / 2}\left(B_{m}\right)\right)$ for all $m$ and $p$ given by

$$
p(x, t)=\lim _{m \rightarrow \infty} \bar{p}^{m}(x, t),
$$

where $\bar{p}^{m}(x, t)$ is defined on $B_{2^{m}}(0)$ by

$$
\bar{p}^{m}(x, t)=-\frac{1}{3}|u(x, t)|^{2}+\text { p.v. } \int_{B_{2}} K_{i j}(x-y) u_{i} u_{j}(y, t) d y+\bar{p}_{3}^{m}+\bar{p}_{4}^{m},
$$

with

$$
\begin{aligned}
& \bar{p}_{3}^{m}(x, t)=\text { p.v. } \int_{B_{2^{m+1} \backslash B_{2}}}\left(K_{i j}(x-y)-K_{i j}(-y)\right) u_{i} u_{j}(y, t) d y \\
& \bar{p}_{4}^{m}(x, t)=\int_{B_{2^{m+1}}^{c}}\left(K_{i j}(x-y)-K_{i j}(-y)\right) u_{i} u_{j}(y, t) d y .
\end{aligned}
$$

To prove this one adapts the argument concerning the convergence of the pressure in $[2$, Proof of Theorem 1.3].

We now establish the local pressure expansion. Following the analogous argument in [2], it is possible to show that

$$
G_{i j}^{Q}\left(u_{i}^{(k)} u_{i}^{(k)}\right) \rightarrow G_{i j}^{Q}\left(u_{i} u_{j}\right) \quad \text { in } L^{3 / 2}\left(0, T ; L^{3 / 2}(Q)\right),
$$

for any cube $Q$ and time $T>0$. Fix a cube $Q$ and $T>0$. Using (4.1) and taking the limit of the weak form of (1.1) we find that the pair $\left(u, G_{i j}^{Q}\left(u_{i} u_{j}\right)\right)$ solves $(1.1)$ in $Q \times(0, T)$. Hence, $\left.\nabla p=\nabla G_{i j}^{Q}\left(u_{i} u_{j}\right)\right)$ in $\mathcal{D}^{\prime}\left(\mathbb{R}^{3}\right)$ at every time $t$ and so there exists a constant $p_{Q}(t)$ so that

$$
\left.p(x, t)=G_{i j}^{Q}\left(u_{i} u_{j}\right)\right)(x, t)+p_{Q}(t),
$$

for $x \in Q$. Clearly we have $p_{Q} \in L^{3 / 2}(0, T)$. This gives the desired local pressure expansion.

At this point we have established the items 1, 2, and 6 from the definition of local energy solutions. The remaining items follow from the arguments in [2] (see also [16, pp. 156-158] and $[19, \S 3])$. This is because for any time $T_{0}$, we have the same convergences of $u^{k}$ and $p^{k}$ on $B_{n} \times T_{0}$ for all $n \in \mathbb{N}$ as in [2]; cf. also [5].

\section{Eventual regularity}

In this section we use Remark 3.5 to prove Theorem 1.4, but first recall a variant of the Caffarelli-Kohn-Nirenberg regularity criteria [7] due to Lin [25]; see also [20, 17].

Lemma 5.1 ( $\epsilon$-regularity criteria). For any $\sigma \in(0,1)$, there exists a universal constant $\epsilon_{*}=\epsilon_{*}(\sigma)>0$ such that, if a pair $(u, p)$ is a suitable weak solutions of (1.1) in $Z_{r}=$ $Z_{r}\left(x_{0}, t_{0}\right)=B_{r}\left(x_{0}\right) \times\left(t_{0}-r^{2}, t_{0}\right)$, and

$$
\epsilon^{3}=\frac{1}{r^{2}} \int_{Z_{r}}\left(|u|^{3}+|p|^{3 / 2}\right) d x d t<\epsilon_{*},
$$

then $u \in L^{\infty}\left(Z_{\sigma r}\right)$. Moreover,

$$
\left\|\nabla^{k} u\right\|_{L^{\infty}\left(Z_{\sigma r}\right)} \leq C_{k} \epsilon r^{-k-1}, \quad k \in \mathbb{N}_{0}
$$

for universal constants $C_{k}=C_{k}(\sigma)$. 
The usual version is $\sigma=1 / 2$. The general version $\sigma \in(0,1)$ follows from the usual version and a partition argument.

Proof of Theorem 1.4. Choose $N_{1}$ such that $\left\|u_{0}\right\|_{M_{\mathcal{C}_{n}}^{2,1}}^{2}<1$ for all $n \geq N_{1}$. Let

$$
Q=Q_{n-1}=\left(-2^{n}, 2^{n}\right)^{3}
$$

Note that $Q \in \mathcal{C}_{n}$ by (1.4). By Lemmas 3.1 and removing the $\log$ factor due to $|Q|^{1 / 3}=2^{n+1}$,

$$
\begin{aligned}
J= & \frac{1}{|Q|^{2 / 3}} \int_{0}^{t} \int_{Q}\left(|u|^{3}+\left|p-p_{Q}(s)\right|^{3 / 2}\right) d x d s \\
\leq & \sup _{Q^{\prime} \in \mathcal{C}_{n} ; Q^{\prime} \cap Q^{* *} \neq \emptyset} \frac{C}{\left|Q^{\prime}\right|^{2 / 3}} \int_{0}^{t} \int_{Q^{\prime}}|u|^{3} d x d s \\
& \quad+C|Q|^{\frac{q}{2}-\frac{7}{6}} \int_{0}^{t}\left(\sup _{Q^{\prime} \in \mathcal{C}_{n}} \frac{1}{\left|Q^{\prime}\right|^{q / 3}} \int_{Q^{\prime}}|u(x, s)|^{2} d x\right)^{3 / 2} d s .
\end{aligned}
$$

Using Lemma 3.2 with $\varepsilon=1$,

$$
\begin{aligned}
J \leq \sup _{Q^{\prime} \in \mathcal{C}_{n} ; Q^{\prime} \cap Q^{* *} \neq \emptyset}|Q|^{q-5 / 3} \int_{0}^{t}\left(\frac{1}{|Q|^{q / 3}} \int_{Q}|u|^{2} d x\right)^{3} d s \\
+|Q|^{-1 / 3} \sup _{Q^{\prime} \in \mathcal{C}_{n} ; Q^{\prime} \cap Q^{* *} \neq \emptyset} \int_{0}^{t} \int_{Q}|\nabla u|^{2} d x d s \\
+C \sup _{Q^{\prime} \in \mathcal{C}_{n} ; Q^{\prime} \cap Q^{* *} \neq \emptyset}|Q|^{\frac{q}{2}-\frac{7}{6}} \int_{0}^{t}\left(\frac{1}{|Q|^{q / 3}} \int_{Q}|u|^{2} d x\right)^{3 / 2} d s \\
+C|Q|^{\frac{q}{2}-\frac{7}{6}} \int_{0}^{t}\left(\sup _{Q^{\prime} \in \mathcal{C}_{n}} \frac{1}{\left|Q^{\prime}\right|^{q / 3}} \int_{Q^{\prime}}|u(x, s)|^{2} d x\right)^{3 / 2} d s .
\end{aligned}
$$

Since the first three terms are the supremum over a finite number (independent of $n$ ) of cubes, we may move the supremum inside the integrals. Taking $q=1$, we get

$$
\begin{aligned}
J \leq & \frac{C}{|Q|^{2 / 3}} \int_{0}^{t}\left(\sup _{Q^{\prime} \in \mathcal{C}_{n}} \frac{1}{\left|Q^{\prime}\right|^{1 / 3}} \int_{Q^{\prime}}|u|^{2} d x\right)^{3} d s+\frac{C}{|Q|^{2 / 3}} \int_{0}^{t}\left(\sup _{Q^{\prime} \in \mathcal{C}_{n}} \frac{1}{\left|Q^{\prime}\right|^{1 / 3}} \int_{Q^{\prime}}|u|^{2} d x\right)^{3 / 2} d s \\
& +\frac{C}{|Q|^{1 / 3}} \sup _{Q^{\prime} \in \mathcal{C}_{n}} \int_{0}^{t} \int_{Q^{\prime}}|\nabla u|^{2} d x d s .
\end{aligned}
$$

Then, using Remark 3.5, $\left\|u_{0}\right\|_{M_{\mathcal{C}_{n}}^{2,1}}^{2} \leq 1$, and taking $t=\bar{T}_{n}=c_{*} 2^{2 n}$ where $c_{*} \in(0,1]$ is the suppressed constant in (3.7), we obtain

$$
\frac{1}{|Q|^{2 / 3}} \int_{0}^{\bar{T}_{n}} \int_{Q}\left(|u|^{3}+\left|p-p_{Q}(s)\right|^{3 / 2}\right) d x d s \leq C\left\|u_{0}\right\|_{M_{\mathcal{C}_{n}}^{2,1}}^{2}
$$

for $n \geq N_{1}$.

We now prove Theorem 1.4. We may assume $0<\delta<1$. Define $\sigma \in(0,1)$ by

$$
\sigma^{2}=\left(1+\frac{\delta}{4}\right)^{-1} \in\left(\frac{4}{5}, 1\right)
$$


By $c_{*} \leq 1$, we have

$$
B_{\sqrt{c_{*}} 2^{n}}(0) \times\left[0, c_{*} 2^{2 n}\right] \subset Q_{n-1} \times\left[0, c_{*} 2^{2 n}\right],
$$

for large $n$. Since $\left\|u_{0}\right\|_{M_{\mathcal{C}_{n}}^{2,1}}^{2} \rightarrow 0$, by (5.2), there exists $N_{2} \geq N_{1}$ so that if $n \geq N_{2}$, then

$$
\frac{1}{c_{*} 2^{2 n}} \int_{0}^{c_{*} 2^{2 n}} \int_{B_{\sqrt{c *} 2^{n}}(0)}\left(|u|^{3}+\left|p-p_{Q}\right|^{3 / 2}\right) d x d t<\frac{\epsilon_{*}(\sigma)}{4} .
$$

By Lemma 5.1, $u$ is regular in

$$
Z_{n}=B_{\sigma \sqrt{c_{*}} 2^{n}}(0) \times\left[\left(1-\sigma^{2}\right) c_{*} 2^{2 n}, c_{*} 2^{2 n}\right]
$$

and $\|u\|_{L^{\infty}\left(Z_{n}\right)} \leq C 2^{-n}$. Note that $Z_{n}$ contains, using (5.3),

$$
P_{n}=\left\{(x, t) \in \mathbb{R}_{+}^{3+1}: \delta|x|^{2} \leq t, \quad\left(1-\sigma^{2}\right) c_{*} 2^{2 n} \leq t \leq 4\left(1-\sigma^{2}\right) c_{*} 2^{2 n}\right\} .
$$

Hence, $u$ is regular in

$$
\bigcup_{n \geq N_{2}} P_{n}=\left\{(x, t) \in \mathbb{R}_{+}^{3+1}: \max \left\{\tau, \delta|x|^{2}\right\} \leq t\right\}, \quad \tau=\left(1-\sigma^{2}\right) c_{*} 2^{2 N_{2}} .
$$

This proves Theorem 1.4.

Remark 5.2. In order to apply Lemma 5.1, we hope to bound both terms on the right side of (5.1) by $\left\|u_{0}\right\|_{M_{\mathcal{C}_{n}}^{2, q}}^{2}$. Anticipating $t \sim|Q|^{2 / 3}$, we need for the second term

$$
\frac{q}{2}-\frac{7}{6}+\frac{2}{3} \leq 0
$$

i.e., $q \leq 1$. Thus $q=1$ is the largest $q$ we may choose to obtain the eventual regularity with our method.

\section{Acknowledgments}

ZB was supported in part by the Simons Foundation, IK was supported in part by the NSF grant DMS-1907992, while TT was supported in part by NSERC grant 261356-18.

\section{References}

[1] A. Basson, Solutions spatialement homogénes adaptées au sens de Caffarelli, Kohn et Nirenberg des équations de Navier-Stokes, Thèse, Université d'Évry, 2006.

[2] Z. Bradshaw and I. Kukavica, Existence of suitable weak solutions to the Navier-Stokes equations for intermittent data, J. Math. Fluid Mech. (to appear).

[3] Z. Bradshaw and T.-P. Tsai, Forward discretely self-similar solutions of the Navier-Stokes equations II, Ann. Henri Poincaré 18 (2017), no. 3, 1095-1119.

[4] Z. Bradshaw and T.-P. Tsai, Discretely self-similar solutions to the Navier-Stokes equations with data in $L_{\mathrm{loc}}^{2}$ satisfying the local energy inequality, Analysis \& PDE (to appear).

[5] Z. Bradshaw and T.-P. Tsai, Global existence, regularity, and uniqueness of infinite energy solutions to the Navier-Stokes equations, arXiv:1907.00256. 
[6] P. Constantin and C. Foias, Navier-Stokes equations, Chicago Lectures in Mathematics, University of Chicago Press, Chicago, IL, 1988.

[7] L. Caffarelli, R. Kohn, and L. Nirenberg, Partial regularity of suitable weak solutions of the Navier-Stokes equations, Comm. Pure Appl. Math. 35 (1982), no. 6, 771-831.

[8] D. Chae and J. Wolf, Existence of discretely self-similar solutions to the Navier-Stokes equations for initial value in $L_{l o c}^{2}\left(\mathbb{R}^{3}\right)$, Ann. Inst. H. Poincaré Anal. Non Linéaire 35 (2018), no. 4, 1019-1039.

[9] R. Dascaliuc and Z. Grujić, Energy cascades and flux locality in physical scales of the 3D NSE, Comm. Math. Phys. 305 (2011), 199-220.

[10] P.G. Fernández-Dalgo and P.G. Lemarié-Rieusset, Weak solutions for Navier-Stokes equations with initial data in weighted $L^{2}$ spaces, arxiv:1906.11038.

[11] G.P. Galdi, An introduction to the Navier-Stokes initial-boundary value problem, Fundamental directions in mathematical fluid mechanics, Adv. Math. Fluid Mech., Birkhäuser, Basel, 2000, pp. 1-70.

[12] Z. Grujić, Regularity of forward-in-time self-similar solutions to the 3D NSE, Discrete Contin. Dyn. Syst. 14 (2006), 837-843.

[13] H. Jia and V. Šverák, Local-in-space estimates near initial time for weak solutions of the Navier-Stokes equations and forward self-similar solutions, Invent. Math. 196 (2014), no. 1, 233-265.

[14] H. Jia and V. Šverák, Minimal $L^{3}$-initial data for potential Navier-Stokes singularities, SIAM J. Math. Anal. 45 (2013), no. 3, 1448-1459.

[15] K. Kang, H. Miura, and T.-P. Tsai, Short time regularity of Navier-Stokes flows with locally $L^{3}$ initial data and applications, arXiv:1812.10509.

[16] N. Kikuchi and G. Seregin, Weak solutions to the Cauchy problem for the Navier-Stokes equations satisfying the local energy inequality, Nonlinear equations and spectral theory, Amer. Math. Soc. Transl. Ser. 2, vol. 220, Amer. Math. Soc., Providence, RI, 2007, pp. 141-164.

[17] I. Kukavica, On partial regularity for the Navier-Stokes equations, Discrete Contin. Dyn. Syst. 21 (2008), no. 3, 717-728.

[18] I. Kukavica and V. Vicol, On local uniqueness of weak solutions to the Navier-Stokes system with $\mathrm{BMO}^{-1}$ initial datum, J. Dynam. Differential Equations 20 (2008), no. 3, 719-732.

[19] H. Kwon and T.-P. Tsai, Global Navier-Stokes flows for non-decaying initial data with slowly decaying oscillation, arxiv:1811.03249.

[20] O.A. Ladyzhenskaya and G.A. Seregin, On partial regularity of suitable weak solutions to the three-dimensional Navier-Stokes equations, J. Math. Fluid Mech. 1 (1999), no. 4, 356-387.

[21] P. G. Lemarié-Rieusset, Recent developments in the Navier-Stokes problem, Chapman \& Hall/CRC Research Notes in Mathematics, vol. 431, Chapman \& Hall/CRC, Boca Raton, FL, 2002.

[22] P.G. Lemarié-Rieusset, The Navier-Stokes equations in the critical Morrey-Campanato space, Rev. Mat. Iberoam. 23 (2007), no. 3, 897-930.

[23] P. G. Lemarié-Rieusset, The Navier-Stokes problem in the 21st century, CRC Press, Boca Raton, FL, 2016. 
[24] J. Leray, Sur le mouvement d'un liquide visqueux emplissant l'espace, Acta Math. 63 (1934), no. $1,193-248$.

[25] F. Lin, A new proof of the Caffarelli-Kohn-Nirenberg theorem, Comm. Pure Appl. Math. 51 (1998), no. 3, 241-257.

[26] Y. Maekawa, H. Miura, and C. Prange, Local energy weak solutions for the Navier-Stokes equations in the half-space, Comm. Math. Phys. 367 (2019), no. 2, 517-580.

[27] J.C. Robinson, J.L. Rodrigo, and W. Sadowski, The three-dimensional Navier-Stokes equations, Cambridge Studies in Advanced Mathematics, vol. 157, Cambridge University Press, Cambridge, 2016, Classical theory.

[28] W. Rusin and V. Šverák, Minimal initial data for potential Navier-Stokes singularities, J. Funct. Anal. 260 (2011), no. 3, 879-891.

[29] R. Temam, Navier-Stokes equations, AMS Chelsea Publishing, Providence, RI, 2001, Theory and numerical analysis, Reprint of the 1984 edition.

[30] T.-P. Tsai, Lectures on Navier-Stokes equations, Graduate Studies in Mathematics, vol. 192, American Mathematical Society, Providence, RI, 2018.

[31] Y. Tsutsui, The Navier-Stokes equations and weak Herz spaces, Adv. Differential Equations 16 (2011), no. 11-12, 1049-1085. 